\title{
Cardiometabolic risk in psoriasis: differential effects of biologic agents
}

\author{
Mariana J Kaplan \\ Department of Internal Medicine, \\ University of Michigan Medical School, \\ Ann Arbor, Michigan, USA
}

\begin{abstract}
Psoriasis is associated to an increased risk of cardiovascular (CV) complications. Overall, the pathogenic mechanisms involved in premature CV complications in psoriasis appear to be complex and multifactorial, with traditional and nontraditional risk factors possibly contributing to the increased risk. Based on what is known about the pathogenesis of psoriasis and extrapolating the current knowledge on CV complications in other inflammatory diseases, studies are needed to investigate if appropriate control of the inflammatory, immunologic and metabolic disturbances present in psoriasis can prevent the development of this potentially lethal complication. It is clear that there is a great need for heightened awareness of the increased risk for vascular damage in patients with psoriasis. It is also crucial to closely monitor patients with psoriasis for $\mathrm{CV}$ risk factors including obesity, hypertension, diabetes, and hyperlipidemia. Whether treatment regimens that effectively manage systemic inflammation will lead to prevention of $\mathrm{CV}$ complications in psoriasis needs to be investigated. Clearly, studies should focus on establishing the exact mechanisms that determine $\mathrm{CV}$ risk in psoriasis so that appropriate preventive strategies and treatment guidelines can be established.
\end{abstract}

Keywords: psoriasis, atherosclerosis, inflammation, vascular

\section{Psoriasis and cardiovascular (CV) disease}

Psoriasis is a chronic inflammatory disorder with an estimated prevalence in the US of $2.2 \%$ in individuals 18 years old and older (Gelfand et al 2005). According to the National Institutes of Health in the US, between 5.8 and 7.5 million Americans have psoriasis. Approximately $6 \%-11 \%$ of patients with psoriasis have an associated inflammatory arthropathy (psoriatic arthritis, PsA). Psoriasis and PsA are characterized by cycles of remission and exacerbation and significantly affect quality of life by causing significant morbidity. Indeed, the impact of psoriasis on health-related quality of life is similar to that of other major medical diseases, including cancer, hypertension and diabetes (Rapp et al 1999).

Similar to other inflammatory disorders, psoriasis is not confined to the skin but, rather, considered a systemic inflammatory condition. This is indicated by several observations, including the presence of elevated inflammatory markers in serum of patients with psoriasis (Rocha-Pereira et al 2004); the development of articular involvement, and the association with the metabolic syndrome (Azfar and Gelfand 2008). Several factors, including genetic determinants, ethnic and regional variations have been associated with disease pathogenesis and with flares of symptomatology (see pathogenesis section below).

Recent evidence indicates that immunological abnormalities in patients with psoriasis may predispose them to an increased risk of other diseases with an inflammatory basis, including atherosclerosis and premature vascular damage. As such, similar to other systemic autoimmune/proinflammatory conditions such as rheumatoid arthritis (RA) (Kaplan, 2006) and systemic lupus erythematosus 
(SLE) (Rajagopalan et al 2004), psoriasis is associated with an increased CV risk. Indeed, the prevalence and severity of coronary artery calcification is significantly higher in patients with psoriasis (Ludwig et al 2007), and several epidemiologic studies have described an association between psoriasis and coronary events, as well as to well-known risk factors of coronary artery disease (CAD) (Gelfand et al 2006; Neimann et al 2006; Shapiro et al 2007; Ludwig et al 2007; Kimball et al 2008). Patients with psoriasis have an increased adjusted relative risk for myocardial infarction (MI) that varies by age. For a 30 -year-old patient with mild or severe psoriasis, relative risk is 1.29 and 3.1 respectively. In a 60 -year-old patient, relative risk is 1.08 for mild psoriasis and 1.36 for severe psoriasis (Gelfand et al 2006). As such, the CV risk conferred by severe psoriasis in young individuals may be comparable to what is seen with other well established risk factors such as diabetes mellitus.

It appears that patients with more diffuse skin involvement have a higher $\mathrm{CV}$ risk. In a large European cohort of patients with psoriasis, the frequency of other co-morbidities associated with increased CV risk (obesity, diabetes mellitus, heart failure and hypertension) was significantly increased when compared to control subjects (Henseler and Christophers 1995). This has been verified by other studies (Shapiro et al 2007). Patients with psoriasis also have altered homocysteine metabolism which may confer additional vascular risk (Malerba et al 2006). The risk of CV death has been reported as $50 \%$ higher in patients hospitalized with psoriasis compared with expected rates(Poikolainen et al 1999). A populationbased study performed in the UK showed that diabetes, hypertension, hyperlipidemia, obesity and smoking are strongly associated with severe psoriasis, when compared to mild psoriasis. However, whether these associations were just the effect of concomitant use of medications potentially linked to increased CV risk factors was not addressed. Psoriasis is also associated with changes in plasma lipids and lipoproteins (Offidani et al 1994; Seishima et al 1994; Uyanik et al 2002) which may play a role in the development of atherosclerotic vascular disease. Increased levels of autoantibodies to oxidized LDL have been reported in patients with psoriasis and may potentially play a role in vascular damage (Orem et al 1999; Sommer et al 2006). Furthermore, patients with psoriasis display elevated markers of inflammatory responses, including C-reactive protein (Strober et al 2008). The strong association between psoriasis and obesity potentially makes the former an important healthcare issue that will likely require an update in its standard of care (Sterry et al 2007; Gisondi et al 2007).
Overall, it is clear that metabolic syndrome prevalence is increased in patients with psoriasis and this could play an important role in heightened CVD development in this disease.

In addition to atherosclerotic $\mathrm{CAD}$, patients with psoriasis appear to be at risk for other CV complications. Arrhythmias are more prevalent in psoriasis (Mastrolonardo 2008). Left ventricular hypertrophy, diastolic dysfunction and valvular pathologies are also more common in this disease (Markuszeski et al 2007). An enhanced stressinduced autonomic response and diminished pituitary adrenal activity have been reported in psoriasis, with higher adrenaline values but diminished cortisol and dehydroepiandrosterone (DHEA) plasma values. This effect decreases with psolaren plus ultraviolet light A (PUVA) treatment (Schmid-Ott et al 1998).

Although psoriatic arthritis may also be associated with an excess prevalence of $\mathrm{CV}$ risk factors, it remains uncertain whether higher rates of clinical CVD are independently associated with the disease itself (reviewed in Roman and Salmon 2007).

Even with a higher prevalence of proatherosclerotic metabolic comorbidities in psoriasis, this disease appears to be an independent risk factor for MI when controlling for major CV risk factors (Gelfand et al 2006). Therefore, the mechanisms contributing to accelerated atherosclerosis and overall CV risk in psoriasis are likely to be multifactorial: a) psoriasis and CVD may share common risk factors, such as tobacco use; b) a higher prevalence of traditional CV risk factors in psoriasis may likely increase CV risk (Cohen et al 2008); c) intrinsic associated risks due to systemic inflammation and immune dysregulation may play a role in vascular damage in psoriasis. This possibility requires confirmation with prospective studies and clearly requires a better understanding of how inflammatory/immune-mediated responses could contribute to vascular damage in this disease.

In order to better understand the potential link of psoriasis with CVD, we will review some of the pathogenic mechanisms recently implicated in the development of psoriasis:

\section{Pathophysiology of psoriasis and PsA}

As no pathogen has been consistently identified within psoriatic plaques, an autoimmune/pro-inflammatory basis has been considered in psoriasis (Nickoloff et al 2007). Overall, psoriasis is characterized by augmented antigen-presenting cell (APC) activation and antigen (Ag) presentation, T-cell activation and Th1 cytokine production. 


\section{Genetic factors}

The precise genetic loci that convey enhanced disease susceptibility in psoriasis remain to be defined. Psoriasis is associated with certain HLA-types (HLA-Cw6, HLA-B13, HLA-B17, HLA-Bw57, and HLA-DR4). People with HLACw6, for example, have a 10-fold higher risk of disease (Schon and Boehncke 2005). The primary locus appears to be located on the short arm of chromosome 6 (PSORS1) within the HLA complex, and appears to be in proximity to HLA-Cw6 (Gudjonsson et al 2006; Lesueur et al 2007). Environmental/epigenetic factors likely play a role in disease pathogenesis, as the concordance in monozygous twins is only $35 \%-56 \%$ (Duffy et al 1993 ).

\section{Cytokines}

Persistent $\mathrm{T}$ cell activation within psoriatic plaques resembles Th1 responses (Hueber and McInnes 2007). TNF- $\alpha$ is overexpressed in psoriatic lesions. NK-T cells and NK cells have also been implicated in the immunopathogenesis of psoriasis and psoriatic arthritis (Chan et al 2003; Gaspari 2006). The T helper (Th) 17 cell, a novel T-cell subset, has been implicated in the pathogenesis of psoriasis and other autoimmune inflammatory diseases (Blauvelt 2008; Haider et al 2008). IL-23 stimulates survival and proliferation of Th17 cells, acting as a key regulator for these diseases. In psoriasis, IL-23 is overproduced by dendritic cells (DCs) and keratinocytes, and this cytokine stimulates Th17 cells within dermis to make IL-17A and IL-22. The latter drives keratinocyte proliferation in psoriasis (Aggarwal et al 2003; Fitch et al 2007). The link between Th1 and Th17 responses in the promotion of CVD is the subject of active investigation (Cheng et al 2008, Wilensky and Hamamdzic, 2007).

\section{Dendritic cells (DCs)}

The interactions between DCs and T-cells, and associated downstream responses play essential roles in the formation of chronic psoriatic plaques (Jariwala 2007). Several abnormalities in DC subsets have been detected within psoriatic plaques. More recently, other DC subsets have been reported within psoriatic skin, including the TNF- $\alpha$ inducible nitric oxide synthases-expressing DCs and the plasmacytoid DCs (Komine et al 2007). The plasmacytoid DCs (pDCs) produce high levels of IFN- $\alpha$ and in animal models of psoriasis, injecting neutralizing antibodies against this molecule prevented the induction of psoriatic lesions after xenotransplantation (Nestle et al 2005). A role for IFN- $\alpha$ in triggering psoriasis is supported by the clinical observations that patients receiving IFN- $\alpha$ injections for different conditions may develop psoriasis or experience a flare of psoriatic lesions (Descamps 2005; Gaal et al 2005; Yurci et al 2007). Interestingly, recent work in SLE has proposed a link between type I IFNs and abnormal vasculogenesis which may promote endothelial dysfunction and, potentially atherosclerosis (Denny et al 2007). Whether type I IFNs play any role in vascular damage in psoriasis remains to be determined.

\section{Other APCs}

Activated macrophages have been linked to the formation of psoriatic plaques (Neylon and Haight 1983; Wang et al 2006). It has been postulated that these cells may circulate from the oral cavity into the skin and trigger a Th1 type cellular immune reaction leading to the formation of psoriatic lesions. A direct role for macrophages in the contribution to cutaneous inflammation was established by the local elimination of macrophages that led to disease resolution (Wang et al 2006). The role of inflammatory macrophages in vascular damage in psoriasis remains to be determined.

\section{T regulatory cells (Tregs)}

While Tregs have been identified in psoriatic lesions (Bovenschen et al 2006), they do not appear to function properly (Kagen et al 2006). Also, 1,25-dihydroxyvitamin D3, an effective therapeutic compound for psoriasis, promotes DC mediated induction of CD4 + Foxp3 + Treg cells (Penna et al 2005). Recent studies from several groups suggest that $\mathrm{T}$ regs may play a role in inhibiting the development and progression of atherosclerosis, adding a potential link between psoriasis and CAD (Gotsman et al 2007).

\section{Infections}

Microorganisms have been associated to the development of psoriasis. $\beta$-hemolytic streptococcal infections are linked to the development of guttate psoriasis and intercurrent infections have been associated to pustular psoriasis flares. Patients with HIV infection have a higher prevalence of psoriasis. However, whether microbial infections play a substantial pathogenic role in the majority of patients with psoriasis has not been demonstrated. Furthermore, whether an infectious agent is involved in vascular damage in psoriasis has not been investigated (Griffiths and Barker 2007).

Overall, shared immunological abnormalities between psoriasis and atherosclerosis include APC and T cell activation, activation of NFKB and Toll-like receptor (TLR) expression, aberrant Tregs and abnormal cytokine responses. Furthermore, both diseases appear to have Th1/Th2 imbalances and increase 
in CD40/CD40 L signaling interaction, upregulation of specific chemokines and adhesion molecules. Activated macrophages are clearly linked to the formation of atherosclerotic plaques involving oxidized forms of LDL. Migratory macrophages and TNF- $\alpha$ are linked to obesity-induced insulin resistance. Several cytokines that have been identified as important mediators of psoriasis, such as IL-1, 4, 6, 8, 12, and TNF- $\alpha$ have also been identified in the metabolic syndrome.

Other mechanisms that have been shown to potentially play a role in premature CVD in other inflammatory conditions such as RA or SLE include cytotoxic T cells (Gerli et al 2004), proinflammatory cytokines (Branen et al 2004), aberrant vascular repair (Grisar et al 2005; Denny et al 2007) and potential genetic influences (reviewed in Kaplan 2006). The role of these factors on the development of CVD in psoriasis has not been studied.

\section{Role of pharmacologic interventions in CV risk in psoriasis}

Several drugs used to treat psoriasis and/or psoriatic arthritis also have effects on the endothelium and, potentially, on the incidence of vascular complications. Extrapolating from what we know about the role of certain immunosuppressants and CV morbidity in RA, there is still considerable uncertainty on the role that certain drugs used to treat psoriasis may play in the health of the endothelium (reviewed in Kaplan 2006). This is in part due to the notion that medications used to treat inflammatory conditions may have dual effects on risk for CV morbidity. For example, steroids might decrease the risk for CV complications in RA by decreasing inflammation, but they might increase it by promoting proatherosclerotic lipid profiles. RA patients exposed to glucocorticoids appear to have an increased incidence of carotid plaque and arterial incompressibility (del Rincon et al 2004). The link between steroids and CVD in SLE is unclear and recent studies suggest a protective effect (Roman et al 2003).

Methotrexate can promote hyperhomocysteinemia (which is corrected by folic acid addition) and cause endothelial damage (Merkle et al 2000; Dierkes and Westphal 2005). However, MTX reduced the incidence of vascular disease in veterans with psoriasis and RA (Choi et al 2002; Prodanovich et al 2005). Low to moderate cumulative doses appeared to be more beneficial than higher doses. In addition, a combination of methotrexate and folic acid led to a further reduction in the incidence of CVD.

The effects of TNF- $\alpha$ blockers on CVD are also complex because these drugs may promote heart failure and decrease heart compliance while controlling inflammation and decreasing risk for plaque formation. Infliximab appears to improve endothelial function in RA after 3 months of therapy
(Hurlimann et al 2002). A Scandinavian study suggested that the risk for developing first $\mathrm{CV}$ events in RA is lower in patients treated with TNF blockers (Jacobsson et al 2005). While this study did not control for most of the traditional and nontraditional risk factors, these results indicate that prospective, long-term, longitudinal studies are required to evaluate the precise role of anti-TNF therapy in atherosclerosis prevention in specific conditions such as psoriasis.

Anti-TNF therapy can also improve other risk factors for accelerated atherosclerosis, including promoting a decrease in insulin resistance, CRP and IL-6, and an increase in high-density lipoprotein (Kiortsis et al 2005; Popa et al 2005). Of note, the beneficial effects of anti-TNF agents on endothelial function do not appear to be sustained and are not seen in diabetic patients treated with etanercept (Gonzalez-Juanatey et al 2004; Dominguez et al 2005). In fact, while an initial improvement in RA endothelial function was observed with infliximab, values returned to baseline 4 weeks after the infusion in patients followed for 1 year (Gonzalez-Juanatey et al 2004). As a cautionary note, while infliximab induces a transient increase in flow-mediated dilatation (FMD), it can also induce vasoconstriction, increases in wall shear stress, and deleterious effects on high-density lipoprotein (Irace et al 2004). It is possible that different TNF antagonists show differential effects on endothelial and smooth muscle cells and on vascular function. Further, animal studies suggest a negative influence of TNF- $\alpha$ inhibitors on collateral artery growth (Grundmann et al 2005). This observation deserves further investigation into the potential role of TNF blockers in decreasing mechanisms of cardiac perfusion in individuals with impaired coronary circulation. Interestingly, in psoriasis, etanercept has been shown to improve insulin sensitivity (Marra et al 2007).

Regarding anti-inflammatory therapies, recent studies indicate that NSAIDs might have a role in suppressing MI risk in RA and other inflammatory conditions and that, ideally, abrupt discontinuation of NSAIDS in these patient populations should be avoided (Fischer et al 2004).

Finally, it appears that statins can mediate modest but clinically apparent anti-inflammatory effects in inflammatory arthritis (McCarey et al 2004). Atorvastatin and simvastatin promoted significant improvements in markers of $\mathrm{CV}$ risk. Recent studies have proposed a beneficial effect of statins in psoriasis activity and their role in CVD prevention in this disease deserves further investigation (Shirinsky and Shirinsky 2007).

\section{Conclusion}

The pathogenic mechanisms involved in accelerated $\mathrm{CV}$ complications in psoriasis and PsA are complex and multifactorial. Overall, studies published over the past 
Table I Factors associated with metabolic abnormalities in systemic inflammatory diseases

\begin{tabular}{lll}
\hline Disease & Risk factor & Preventive strategy \\
\hline SLE & Steroids & $\begin{array}{l}\text { Minimize prolonged steroid } \\
\text { use (Bultink et al 2008) }\end{array}$ \\
SLE & Active disease & $\begin{array}{l}\text { Adequate control of disease } \\
\text { activity (Sidiropoulos et al 2008) }\end{array}$ \\
SLE & Sedentary life-style & $\begin{array}{l}\text { Exercise (Negron et al 2008) } \\
\text { Adequate control of disease }\end{array}$ \\
RA & Active disease & $\begin{array}{l}\text { activity (Dessein and Joffe 2006) } \\
\text { Normal body mass (Dessein } \\
\text { and Joffe 2006) }\end{array}$ \\
AS & Obesity & Adequate control of disease \\
& Activity (Sidiropoulos et al 2008)
\end{tabular}

Abbreviation: AS, ankylosing spondylitis; RA, rheumatoid arthritis; SLE, systemic lupus erythematosus.

decade have confirmed a higher risk for CV complications in psoriasis, and indicate that aggressive prevention and treatment of risk factors are likely to be of extreme importance in this patient population. Given what we know about the development of metabolic syndrome (Table 1) and CV risk factors in other systemic inflammatory diseases, these strategies will likely include appropriate control of the inflammatory abnormalities and metabolic changes seen in psoriasis. Established and potential preventive strategies are summarized in Table 2. More studies are required to assess the exact role that anti-TNF therapies and other biologicals might have in CVD prevention in psoriasis. It is clear that there is a great need for heightened awareness of the increased risk for vascular damage in patients with psoriasis.

Table 2 Preventive strategies for traditional and non-traditional cardiovascular risk factors in patients with psoriasis

\begin{tabular}{|c|c|}
\hline Risk factor & Preventive strategy \\
\hline Smoking & $\begin{array}{l}\text { Counseling, nicotine patches/gum, } \\
\text { bupropion }\end{array}$ \\
\hline Obesity & Counseling, diet, exercise \\
\hline Hypertension & $\begin{array}{l}\text { Monitoring, diet, exercise, minimize } \\
\text { corticosteroids and NSAIDS; } \\
\text { prompt use of antihypertensives }\end{array}$ \\
\hline Hyperlipidemia & $\begin{array}{l}\text { Monitoring, counseling, diet, } \\
\text { exercise, limit systemic } \\
\text { corticosteroids. }\end{array}$ \\
\hline Insulin resistance & Anti-inflammatories/biologicals \\
\hline Hyperhomocystenemia & $\begin{array}{l}\text { Folic acid supplementation with } \\
\text { methotrexate use }\end{array}$ \\
\hline Family history of CVD & Counseling, monitoring risk factors \\
\hline Inflammation & Anti-inflammatories, biologicals ${ }^{\mathrm{a}}$. \\
\hline Additional prethrombotic risk & $\begin{array}{l}\text { Low dose aspirin/consider } \\
\text { anticoagulation. }\end{array}$ \\
\hline
\end{tabular}

aHypothetical. Requires confirmation with controlled studies.

\section{Disclosures}

The author has no conflicts of interest to disclose.

\section{References}

Aggarwal S, Ghilardi N, Xie MH, et al. 2003. Interleukin-23 promotes a distinct CD4 $\mathrm{T}$ cell activation state characterized by the production of interleukin-17. J Biol Chem, 278:1910-4.

Azfar RS, Gelfand JM. 2008. Psoriasis and metabolic disease: epidemiology and pathophysiology. Curr Opin Rheumatol, 20:416-22.

Blauvelt A. 2008. T-helper 17 cells in psoriatic plaques and additional genetic links between IL-23 and psoriasis. J Invest Dermatol, 128:1064-7.

Bovenschen HJ, Van Vlijmen-Willems IM, Van De Kerkhof PC. et al. 2006. Identification of lesional CD4 + CD25 + Foxp3 + regulatory T cells in psoriasis. Dermatology, 213:111-7.

Branen L, Hovgaard L, Nitulescu M, et al. 2004. Inhibition of tumor necrosis factor-alpha reduces atherosclerosis in apolipoprotein $\mathrm{E}$ knockout mice. Arterioscler Thromb Vasc Biol, 24:2137-42.

Chan WL, Pejnovic N, Liew TV et al. 2003. NKT cell subsets in infection and inflammation. Immunol Lett, 85:159-63.

Cheng X, Yu X, Ding YJ, et al. 2008. The Th17/Treg imbalance in patients with acute coronary syndrome. Clin Immunol, 127:89-97.

Choi HK, Hernan MA, Seeger JD, et al. 2002. Methotrexate and mortality in patients with rheumatoid arthritis: a prospective study. Lancet, 359:1173-7.

Cohen AD, Sherf M, Vidavsky L, et al. 2008. Association between psoriasis and the metabolic syndrome. A cross-sectional study. Dermatology, 216:152-5.

Del Rincon I, O’Leary DH, Haas RW, et al. 2004. Effect of glucocorticoids on the arteries in rheumatoid arthritis. Arthritis Rheum, 50:3813-22.

Denny MF, Thacker S, Mehta H, et al. 2007. Interferon-alpha promotes abnormal vasculogenesis in lupus: a potential pathway for premature atherosclerosis. Blood, 110:2907-15.

Descamps V. 2005. [Cutaneous side effects of alpha interferon]. Presse Med, 34:1668-72.

Dierkes J, Westphal S. 2005. Effect of drugs on homocysteine concentrations. Semin Vasc Med, 5:124-39.

Dominguez H, Storgaard H., Rask-Madsen C, et al. 2005. Metabolic and vascular effects of tumor necrosis factor-alpha blockade with etanercept in obese patients with type 2 diabetes. $J$ Vasc Res, 42:517-25.

Duffy DL, Spelman LS, Martin NG. 1993. Psoriasis in Australian twins. J Am Acad Dermatol, 29:428-34.

Fischer LM, Schlienger RG, Matter CM, et al. 2004. Discontinuation of nonsteroidal anti-inflammatory drug therapy and risk of acute myocardial infarction. Arch Intern Med, 164:2472-6.

Fitch E, Harper E, Skorcheva I, et al. 2007. Pathophysiology of psoriasis:recent advances on IL-23 and Th17 cytokines. Curr Rheumatol Rep, 9:461-7.

Gaal J, Kalmanchey J, Szegedi A, et al. 2005. [Interferon induced psoriatic arthritis in a patient with renal cancer]. Orv Hetil, 146:2503-6.

Gaspari AA. 2006. Innate and adaptive immunity and the pathophysiology of psoriasis. J Am Acad Dermatol, 54:S67-80.

Gelfand JM, Neimann AL, Shin DB, et al. 2006. Risk of myocardial infarction in patients with psoriasis. Jama, 296:1735-41.

Gelfand JM, Weinstein R, Porter SB, et al. 2005. Prevalence and treatment of psoriasis in the United Kingdom: a population-based study. Arch Dermatol, 141:1537-41.

Gerli R, Schillaci G, Giordano A, et al. 2004. CD4 + CD28- T lymphocytes contribute to early atherosclerotic damage in rheumatoid arthritis patients. Circulation, 109:2744-8.

Gisondi P, Tessari G, Conti A, et al. 2007. Prevalence of metabolic syndrome in patients with psoriasis: a hospital-based case-control study. Br J Dermatol, 157:68-73.

Gonzalez-Juanatey C, Testa A, Garcia-Castelo A, et al. 2004. Active but transient improvement of endothelial function in rheumatoid arthritis patients undergoing long-term treatment with anti-tumor necrosis factor alpha antibody. Arthritis Rheum, 51:447-50. 
Gotsman I, Gupta R, Lichtman AH. 2007. The influence of the regulatory T lymphocytes on atherosclerosis. Arterioscler Thromb Vasc Biol, 27:2493-5.

Griffiths CE, Barker JN. 2007. Pathogenesis and clinical features of psoriasis. Lancet, 370:263-71.

Grisar J, Aletaha D, Steiner CW, et al. 2005. Depletion of endothelial progenitor cells in the peripheral blood of patients with rheumatoid arthritis. Circulation, 111:204-11.

Grundmann S, Hoefer I, Ulusans S, et al. 2005. Anti-tumor necrosis factor-\{alpha $\}$ therapies attenuate adaptive arteriogenesis in the rabbit. Am J Physiol Heart Circ Physiol, 289:H1497-505.

Gudjonsson JE, Karason A, Runarsdottir EH, et al. 2006. Distinct clinical differences between HLA-Cw*0602 positive and negative psoriasis patients-an analysis of 1019 HLA-C- and HLA-B-typed patients. J Invest Dermatol, 126:740-5.

Haider AS, Lowes MA, Suarez-Farinas M, et al. 2008. Identification of cellular pathways of "type 1," Th17 T cells, and TNF- and inducible nitric oxide synthase-producing dendritic cells in autoimmune inflammation through pharmacogenomic study of cyclosporine A in psoriasis. J Immunol, 180:1913-20.

Henseler T, Christophers E. 1995. Disease concomitance in psoriasis. $J$ Am Acad Dermatol, 32:982-6.

Hueber AJ, McInnes IB. 2007. Immune regulation in psoriasis and psoriatic arthritis-recent developments. Immunol Lett, 114:59-65.

Hurlimann D, Forster A, Noll G, et al. 2002. Anti-tumor necrosis factor-alpha treatment improves endothelial function in patients with rheumatoid arthritis. Circulation, 106:2184-7.

Irace C, Mancuso G, Fiaschi E, et al. 2004. Effect of anti TNFalpha therapy on arterial diameter and wall shear stress and HDL cholesterol. Atherosclerosis, 177:113-8.

Jacobsson LT, Turesson C, Gulfe A, et al. 2005. Treatment with tumor necrosis factor blockers is associated with a lower incidence of first cardiovascular events in patients with rheumatoid arthritis. J Rheumatol, 32:1213-8.

Jariwala SP. 2007. The role of dendritic cells in the immunopathogenesis of psoriasis. Arch Dermatol Res, 299:359-66.

Kagen MH, McCormick TS, Cooper KD. 2006. Regulatory T cells in psoriasis. Ernst Schering Res Found Workshop. p193-209.

Kaplan MJ. 2006. Cardiovascular disease in rheumatoid arthritis. Curr Opin Rheumatol, 18:289-97.

Kimball AB, Robinson D Jr, Wu Y, et al. 2008. Cardiovascular disease and risk factors among psoriasis patients in two US healthcare databases, 2001-2002. Dermatology, 217:27-37.

Kiortsis DN, Mavridis AK, Vasakos S, et al. 2005. Effects of infliximab treatment on insulin resistance in patients with rheumatoid arthritis and ankylosing spondylitis. Ann Rheum Dis, 64:765-6.

Komine M, Karakawa M, Takekoshi T, et al. 2007. Early inflammatory changes in the "perilesional skin"of psoriatic plaques:is there interaction between dendritic cells and keratinocytes? J Invest Dermatol, 127:1915-22.

Lesueur F, Lefevre C, Has C, et al. 2007. Confirmation of psoriasis susceptibility loci on chromosome 6p21 and 20p13 in French families. J Invest Dermatol, 127:1403-9.

Ludwig RJ, Herzog C, Rostock A et al. 2007. Psoriasis: a possible risk factor for development of coronary artery calcification. Br J Dermatol, 156:271-6.

McCarey DW, McInnes IB, Madhok R, et al. 2004. Trial of Atorvastatin in Rheumatoid Arthritis (TARA): double-blind, randomised placebocontrolled trial. Lancet, 363:2015-21.

Malerba M, Gisondi P, Radaeli A, et al. 2006. Plasma homocysteine and folate levels in patients with chronic plaque psoriasis. Br J Dermatol, 155:1165-9.

Markuszeski L, Bissinger A, Janusz I, et al. 2007. Heart rate and arrhythmia in patients with psoriasis vulgaris. Arch Med Res, 38:64-9.

Marra M, Campanati A, Testa R, et al. 2007. Effect of etanercept on insulin sensitivity in nine patients with psoriasis. Int J Immunopathol Pharmacol, 20:731-6.
Mastrolonardo M. 2008. Sympathetic nervous system dysfunction: a common pathway linking skin and heart morbidity? $J$ Am Acad Dermatol, $58: 352$

Merkle CJ, Moore IM, Penton BS, et al. 2000. Methotrexate causes apoptosis in postmitotic endothelial cells. Biol Res Nurs, 2:5-14.

Neimann AL, Shin DB, Wang X, et al. 2006. Prevalence of cardiovascular risk factors in patients with psoriasis. $J$ Am Acad Dermatol, 55:829-35.

Nestle FO, Conrad C, Tun-Kyi A, et al. 2005. Plasmacytoid predendritic cells initiate psoriasis through interferon-alpha production. J Exp Med, 202:135-43.

Neylon L, Haight JR. 1983. Neocortical projections of the suprageniculate and posterior thalamic nuclei in the marsupial brush-tailed possum, Trichosurus vulpecula (Phalangeridae), with a comparative commentary on the organization of the posterior thalamus in marsupial and placental mammals. J Comp Neurol, 217:357-75.

Nickoloff BJ, Qin JZ, Nestle FO. 2007. Immunopathogenesis of psoriasis. Clin Rev Allergy Immunol, 33:45-56.

Offidani AM, Ferretti G, Taus M, et al. 1994. Lipoprotein peroxidation in adult psoriatic patients. Acta Derm Venereol Suppl (Stockh), 186:38-40.

Orem A, Cimsit G, Deger O, et al. 1999. The significance of autoantibodies against oxidatively modified low-density lipoprotein (LDL) in patients with psoriasis. Clin Chim Acta, 284:81-8.

Penna G, Roncari A, Amuchastegui S, et al. 2005. Expression of the inhibitory receptor ILT3 on dendritic cells is dispensable for induction of CD4 + Foxp3 + regulatory T cells by 1,25-dihydroxyvitamin D3. Blood, 106:3490-7.

Poikolainen K, Karvonen J, Pukkala E. 1999. Excess mortality related to alcohol and smoking among hospital-treated patients with psoriasis. Arch Dermatol, 135:1490-3.

Popa C, Netea MG, Radstake T, et al. 2005. Influence of anti-tumour necrosis factor therapy on cardiovascular risk factors in patients with active rheumatoid arthritis. Ann Rheum Dis, 64:303-5.

Prodanovich S, Ma F, Taylor JR, et al. 2005. Methotrexate reduces incidence of vascular diseases in veterans with psoriasis or rheumatoid arthritis. $J$ Am Acad Dermatol, 52:262-7.

Rajagopalan S, Somers EC, Brook RD, et al. 2004. Endothelial cell apoptosis in systemic lupus erythematosus: a common pathway for abnormal vascular function and thrombosis propensity. Blood, 103:3677-83.

Rapp SR, Feldman SR, Exum ML, et al. 1999. Psoriasis causes as much disability as other major medical diseases. $J$ Am Acad Dermatol, 41:401-7.

Rocha-Pereira P, Santos-Silva A, Rebelo I, et al. 2004. The inflammatory response in mild and in severe psoriasis. Br J Dermatol, 150:917-28.

Roman MJ, Salmon JE. 2007. Cardiovascular manifestations of rheumatologic diseases. Circulation, 116:2346-55.

Roman MJ, Shanker BA, Davis A, et al. 2003. Prevalence and correlates of accelerated atherosclerosis in systemic lupus erythematosus. $N$ Engl J Med, 349:2399-406.

Schmid-Ott G, Jacobs R, Jager B, et al. 1998. Stress-induced endocrine and immunological changes in psoriasis patients and healthy controls. A preliminary study. Psychother Psychosom, 67:37-42.

Schon MP, Boehncke WH. 2005. Psoriasis. N Engl J Med, 352:1899-912.

Seishima M, Seishima M, Mori S, et al. 1994. Serum lipid and apolipoprotein levels in patients with psoriasis. Br J Dermatol, 130:738-42.

Shapiro J, Cohen AD, David M, et al. 2007. The association between psoriasis, diabetes mellitus, and atherosclerosis in Israel: a case-control study. J Am Acad Dermatol, 56:629-34.

Shirinsky IV, Shirinsky VS. 2007. Efficacy of simvastatin in plaque psoriasis:A pilot study. $J$ Am Acad Dermatol, 57:529-31.

Sommer DM, Jenisch S, Suchan M, et al. 2006. Increased prevalence of the metabolic syndrome in patients with moderate to severe psoriasis. Arch Dermatol Res, 298:321-8.

Sterry W, Strober BE, Menter A. 2007. Obesity in psoriasis: the metabolic, clinical and therapeutic implications. Report of an interdisciplinary conference and review. Br J Dermatol, 157:649-55. 
Strober B, Teller C, Yamauchi P, et al. 2008. Effects of etanercept on C-reactive protein levels in psoriasis and psoriatic arthritis. Br J Dermatol. In press.

Uyanik BS, Ari Z, Onur E, et al. 2002. Serum lipids and apolipoproteins in patients with psoriasis. Clin Chem Lab Med, 40:65-8

Wang H, Peters T, Kess D, et al. 2006. Activated macrophages are essential in a murine model for $\mathrm{T}$ cell-mediated chronic psoriasiform skin inflammation. J Clin Invest, 116:2105-14.
Wilensky RL, Hamamdzic D. 2007. The molecular basis of vulnerable plaque: potential therapeutic role for immunomodulation. Curr Opin Cardiol, 22:545-51.

Yurci A, Guven K, Torun E, et al. 2007. Pyoderma gangrenosum and exacerbation of psoriasis resulting from pegylated interferon alpha and ribavirin treatment of chronic hepatitis C. Eur J Gastroenterol Hepatol, 19:811-5. 
